EPJ Web of Conferences 71, 00021 (2014)

DOI: 10.1051/epjconf/20147100021

(C) Owned by the authors, published by EDP Sciences, 2014

\title{
Overview of $\mathrm{LHCb}$ results on $C P$ violation
}

\author{
Markward Britsch ${ }^{1, a}$ (on behalf of the LHCb Collaboration) \\ ${ }^{1}$ Max-Planck-Institut für Kernphysik, Saupfercheckweg 1, 69117 Heidelberg, Germany
}

\begin{abstract}
Precision measurements of $C P$ violating observables in the mixing and decay of $B$ mesons provide excellent opportunities to search for possible contributions from New Physics beyond the Standard Model. In this contribution, key measurements pursued by the LHCb collaboration at CERN's Large Hadron Collider (LHC) are described. Important results have been reported on topics including the $C P$ violating phase $\phi_{s}$ in $B_{s}^{0} \rightarrow J / \psi \phi$ (and related) decays, the angle $\gamma$ of the CKM unitarity triangle, and direct $C P$ violation effects in two- and three-body $B$ meson decays to charmless final states. Latest results on $C P$ violation in the charm sector are also reviewed. Results obtained from the analysis of data collected in 2011 already match or even surpass the measurements of previous experiments in their precision. All results obtained so far are compatible with Standard Model predictions.
\end{abstract}

\section{Introduction}

Why is $C P$ violation of special interest? $C P$ is the combination of the charge conjugation operator $C$ and the parity operator $P$. To put it very simply, it turns particles into anti-particles and vice versa. $C P$ violation, i.e., the violation of symmetry under $C P$, was first observed in the $K^{0}$ system and was an indirect indication for new quarks, directly seen only over a decade later. This is an example of how flavour physics, i.e., the physics of electroweak changes of quark or lepton flavour changes, can be very powerful in finding effects of new heavy particles. An introduction to $C P$ violation and related subjects can be found in, e.g., Refs. [1,2]. $C P$ violation is also needed to explain the matter-antimatter asymmetry in the universe, i.e., the puzzle that the same amount of matter and anti-matter has been created in the Big Bang, although today we observe only matter. In the Standard Model (SM) the $C P$ violation is much too small to accomplish this, thus we expect deviations from the $\mathrm{SM}$ in $C P$ violation.

There are three main ways to measure $C P$ violation. Firstly, direct $C P$ violation can be measured in a decay of a particle $B$ to final state $f$, as it leads to $\Gamma(B \rightarrow f) \neq \Gamma(\bar{B} \rightarrow \bar{f})$, where the bar denotes the $C P$ transformed state and $\Gamma$ is the partial decay width. Experimentally it can be obtained by measuring the asymmetry $A_{C P}^{\mathrm{dir}}=\frac{\Gamma(\bar{B} \rightarrow \bar{f})-\Gamma(B \rightarrow f)}{\Gamma(\bar{B} \rightarrow \bar{f})+\Gamma(B \rightarrow f)}$ or equivalently $A_{C P}^{\mathrm{dir}}=\frac{N_{\bar{f}}-N_{f}}{N_{\tilde{f}}+N_{f}}$ for the total numbers $N_{f}$ and $N_{\bar{f}}$ of measured decays to $f$ and $\bar{f}$ respectively. New Physics (NP) can enter if the Feynman diagram of the decay contains a loop, as in the so called penguin diagrams as depicted in Fig. 1. In this case one of the SM particles in the loop can be replaced by a possibly very heavy NP particle.

ae-mail: Markward.Britsch@mpi-hd.mpg.de

This is an Open Access article distributed under the terms of the Creative Commons Attribution License 2.0, which permits unrestricted use, distribution, and reproduction in any medium, provided the original work is properly cited. 


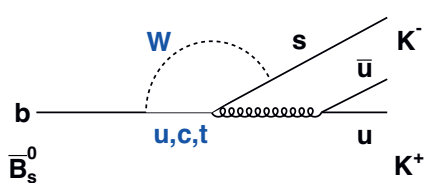

$\overline{\mathbf{s}}$

Figure 1. Example of a Feynman diagram of the kind called penguin diagram. NP particles could replace the SM particles in the loop (in blue).
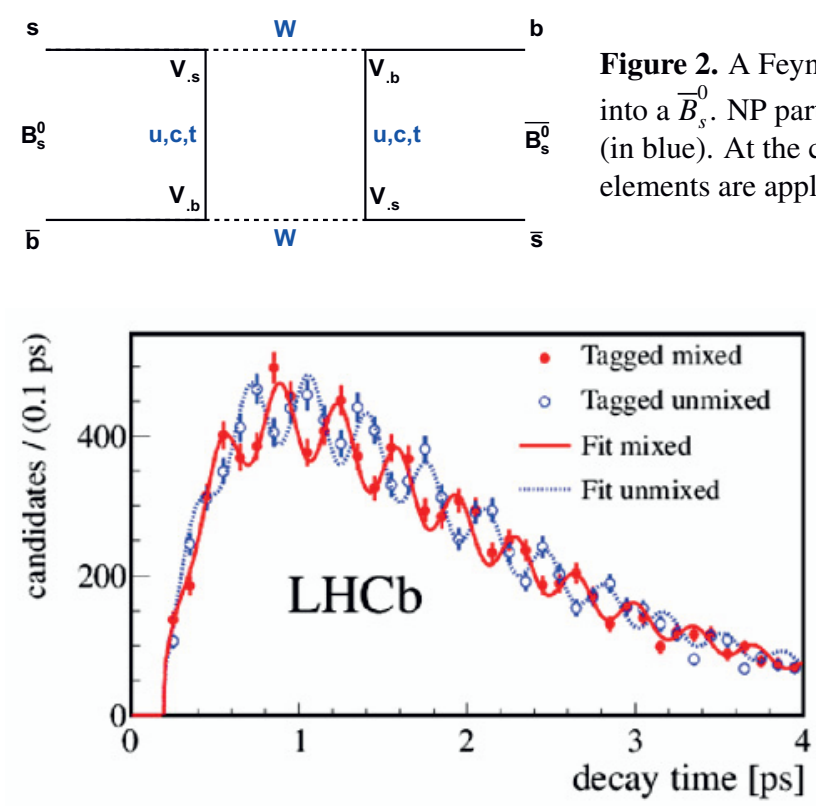

Figure 2. A Feynman diagram that describes the mixing of a $B_{s}^{0}$ into a $\bar{B}_{s}^{0}$. NP particles could replace the SM particles in the box (in blue). At the corners of the box different CKM-matrix elements are applied.

A second way of measuring $C P$ violation is in flavour mixing. The particles $B_{s}^{0}, B^{0}, K^{0}$ and $D^{0}$ have the ability to turn into their anti-particles. One possible Feynman diagram of such a process is shown in Fig. 2. NP could come in, replacing the $u, c, t, W$-propagators in the Feynman diagram. Experimentally, mixing is observed in oscillations where one flavour state is turned into its anti-state with the mixing frequency $\omega=\Delta m$ being equal to the mass difference between the two states in natural units $^{1}$, shown in Fig. 3 for the $B_{s}^{0}$. $C P$ violation leads to unequal probabilities $P\left(B_{s}^{0} \rightarrow \bar{B}_{s}^{0}\right) \neq P\left(\bar{B}_{s}^{0} \rightarrow\right.$ $B_{s}^{0}$ ) and could be observed as a time independent asymmetry between decays into flavour specific final states $A_{C P}^{\mathrm{mix}}=\frac{\Gamma\left(\bar{B}_{s}(t) \rightarrow \bar{f}\right)-\Gamma\left(B_{s}(t) \rightarrow f\right)}{\Gamma\left(\bar{B}_{s}(t) \rightarrow \bar{f}\right)+\Gamma\left(B_{s}(t) \rightarrow f\right)}$, where $B_{s}(t)$ is the state into which a produced $B_{s}$ meson has evolved after a proper time $t$.

In Fig. 2 at each corner of the box, the CKM-matrix

$$
\left(\begin{array}{c}
d^{\prime} \\
s^{\prime} \\
b^{\prime}
\end{array}\right)=\left(\begin{array}{lll}
V_{u d} & V_{u s} & V_{u b} \\
V_{c d} & V_{c s} & V_{c b} \\
V_{t d} & V_{t s} & V_{t b}
\end{array}\right)\left(\begin{array}{c}
d \\
s \\
b
\end{array}\right)
$$

is at work, where $d, s, b$ are the mass eigenstates of the quarks and $d^{\prime}, s^{\prime}, b^{\prime}$ are their weak eigenstates. In the SM, the CKM-matrix is unitary and thus different unitarity conditions can be derived. One of these is that the first column of the matrix multiplied by the complex conjugated of the third column,

\footnotetext{
${ }^{1}$ Natural units have $c=\hbar=1$
} 


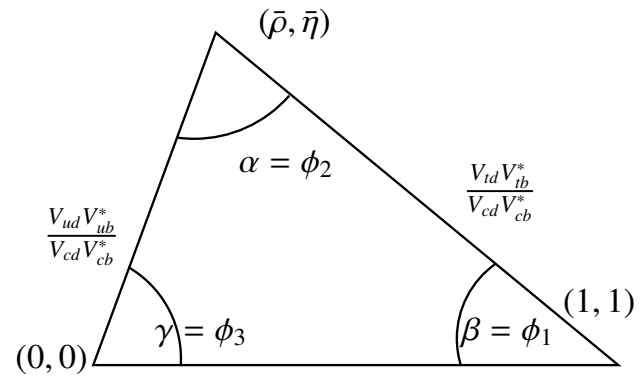

Figure 4. The (normalized) standard unitary triangle. This is a representation of Eq. (1) where the horizontal direction represents the real part while the vertical direction represents the imaginary part of a complex number.

has to be zero:

$$
V_{u d} V_{u b}^{*}+V_{c d} V_{c b}^{*}+V_{t d} V_{t b}^{*}=0 .
$$

This can be interpreted as the standard unitarity triangle, as such an equation describes a triangle in complex space. Figure 4 shows the standard unitary triangle with both nomenclatures of the angles that are in use, and of which we will use $\alpha, \beta, \gamma . C P$ violation occurs in this context if $\bar{\eta} \neq 0$, i.e., if the triangle is not degenerated to a line. To check unitarity (and thus probe the SM) one can test if this is really a triangle, e.g., by testing if the sum of the three angles gives $180^{\circ}$. Of these angles, $\gamma$ is the least well measured one to date and thus experimentally the most interesting one. To measure these angles the (relative) quantum phase of the $C P$ violation effect has to be measured.

The third method to measure $C P$ violation is to measure it in the interference between mixing and decay. To do so, usually a final state $f_{C P}$ is used that is a $C P$ eigenstate. It must be a possible final state of the decaying particle as well as of the anti-particle, i.e., $B_{s}^{0} \rightarrow f_{C P}$ and $B_{s}^{0} \rightarrow \bar{B}_{s}^{0} \rightarrow f_{C P}$, must be possible. To access this experimentally, the time dependent asymmetry

$$
\frac{\Gamma\left(\bar{B}_{s}^{0} \rightarrow f_{C P}, t\right)-\Gamma\left(B_{s}^{0} \rightarrow f_{C P}, t\right)}{\Gamma\left(\bar{B}_{s}^{0} \rightarrow f_{C P}, t\right)+\Gamma\left(B_{s}^{0} \rightarrow f_{C P}, t\right)}=\frac{S_{f_{C P}} \sin (\Delta m t)-C_{f_{C P}} \cos (\Delta m t)}{\cosh \left(\frac{1}{2} \Delta \Gamma t\right)-A_{f_{C P}}^{\Delta \Gamma} \sinh \left(\frac{1}{2} \Delta \Gamma t\right)}
$$

is used, where $\Delta \Gamma$ is the difference between the decay widths of two states, $S_{f_{C P}}$ is the indirect $C P$ violation parameter and $C_{f_{C P}}$ is the direct $C P$ violation parameter. The indirect $C P$ violation parameter is a function of the quantum phase of the mixing $\phi_{m}$ and the quantum phase that appears in the decay $\phi_{D}$, which are both typically functions of unitary triangle angles. NP is again possible in a decay through penguin diagrams and through the box diagrams in the mixing.

The LHCb detector [4] at the LHC is a single arm spectrometer that is optimised for beauty and charm physics, including $C P$ violation measurements. A schematic view is provided in Fig. 5. To resolve the very fast $B_{s}^{0}$ oscillations it has excellent vertex resolution and tracking. The combined tracking system has momentum resolution $\Delta p / p$ that varies from $0.4 \%$ at $5 \mathrm{GeV} / c$ to $0.6 \%$ at 100 $\mathrm{GeV} / c$. Its good particle identification system consists of two Ring Imaging Cherenkov detectors (RICH) that can separate pions from kaons and protons, the electromagnetic calorimeter (ECAL) being able to separate electrons from photons, and the MUON chambers that are able to identify muons. The trigger has two main stages, level 0 is done in hardware triggering on high transverse momentum $\left(p_{\mathrm{T}}\right)$ hadron calorimeter (HCAL), ECAL and MUON objects. The high level trigger is implemented in software and does a global event reconstruction and selection.

The $p p$-data at centre of mass energies $\sqrt{s}=7 \mathrm{TeV}$ and $8 \mathrm{TeV}$ taken by LHCb amounts to an integrated luminosity of $\mathcal{L} \sim 3 \mathrm{fb}^{-1}$ altogether. About $38 \mathrm{pb}^{-1}$ have been taken in 2010 at $\sqrt{s}=7 \mathrm{TeV}$. At the same centre of mass energy about $1 \mathrm{fb}^{-1}$ of data have been recorded in 2011. In 2012 data corresponding to $2 \mathrm{fb}^{-1}$ have been taken at $\sqrt{s}=8 \mathrm{TeV}$. The overall data taking efficiency was $93 \%$. 


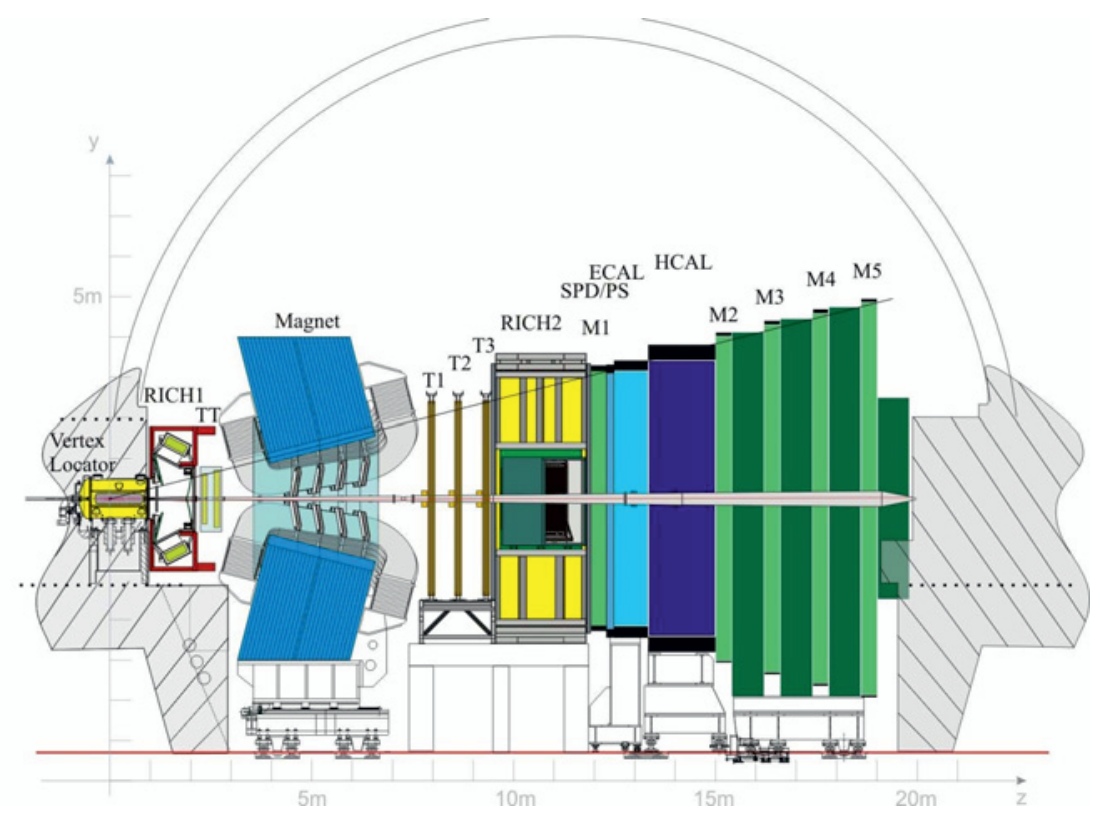

Figure 5. Schematic view of the LHCb detector [4].

Table 1. All decay modes with published results on $C P$ violation by LHCb (as of August 2013). An 'h' stands here for a charged pion or kaon.

\begin{tabular}{lll}
\hline beauty, time independent & beauty, time dependent & charm \\
\hline$B^{+} \rightarrow J / \psi \pi^{+}, \psi(2 S)[5]$ & $B^{0} \rightarrow \pi^{+} \pi^{-}, B_{s}^{0} \rightarrow K^{+} K^{-}[6]$ & $D^{0} \rightarrow h^{+} h^{-}[7,8]$ \\
$B^{+} \rightarrow K_{\mathrm{s}}^{0} h^{+}[9]$ & $B^{0} \rightarrow J / \psi K_{\mathrm{s}}^{0}[10]$ & $D^{+} \rightarrow K_{\mathrm{s}}^{0} \pi^{+}[11]$ \\
$B_{d / s}^{0} \rightarrow K^{+} \pi^{-}[12]$ & $B_{s}^{0} \rightarrow \phi \phi[13]$ & $D^{+} \rightarrow \phi \pi^{+}[11]$ \\
$B^{0} \rightarrow K^{* 0} \mu^{+} \mu^{-}[14]$ & $B_{s}^{0} \rightarrow J / \psi K^{+} K^{-}, J / \psi \pi^{+} \pi^{-}[15]$ & $D^{0} \rightarrow \pi^{+} \pi^{-} \pi^{+} \pi^{-}[16]$ \\
$B^{+} \rightarrow K^{+} \mu^{+} \mu^{-}[17]$ & $B_{s}^{0} \rightarrow D_{s}^{+} K^{-}[18]$ & $D^{0} \rightarrow K^{+} K^{-} \pi^{+} \pi^{-}[16]$ \\
$B^{0} \rightarrow J / \psi K^{* 0}[19]$ & & $D^{+} \rightarrow K^{+} K^{-} \pi^{+}[20]$ \\
$B^{0} \rightarrow K^{* 0} \gamma[21]$ & & \\
$B^{+} \rightarrow K^{+} \pi^{-} \pi^{+} \gamma[22]$ & & \\
$B^{+} \rightarrow D^{0} h^{+}$(measuring $\left.\gamma\right)[23]$ & & \\
$B^{+} \rightarrow h^{+} h^{-} h^{+}[24]$ & & \\
$B^{+} \rightarrow p \bar{p} h^{+}[25]$ & & \\
$B_{s}^{0} \rightarrow D_{s}^{+} X \mu^{-} \bar{v}(C P$ in mixing) [26] & & \\
$B^{0} \rightarrow D^{0} K^{* 0}[27]$ & & \\
$\gamma$ combination [28] & & \\
\hline
\end{tabular}

Table 1 lists all the channels where $C P$ violation has been searched for at LHCb, where ' $h$ ' - as in the remainder of these proceedings - stands for a charged pion or kaon. Charge conjugated processes are always implicitly included throughout these proceedings except where they are explicitly mentioned. 


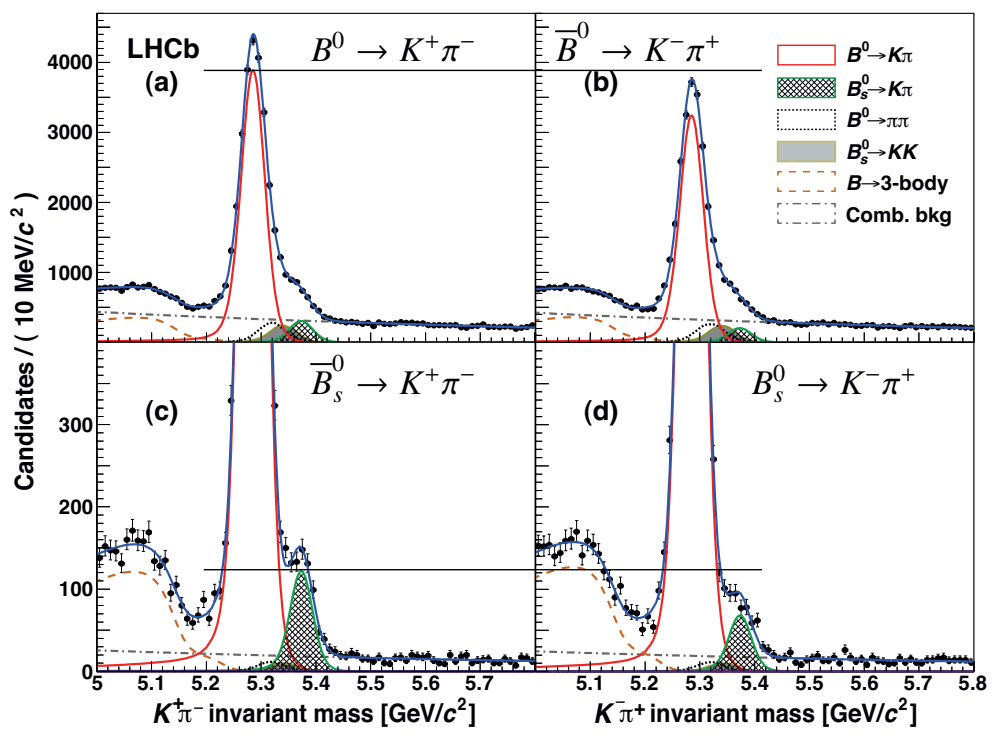

Figure 6. Invariant mass of $K^{+} \pi^{-}$(left) and $K^{-} \pi^{+}$(right) with a selection to optimise the $B^{0}$ signal (top) and one that optimises the $B_{s}^{0}$ signal (bottom) [12]. The black circles with error bars are the data, while the blue line is a fit to them. The red line is the $B^{0}$ signal component of the fit, while the green line (filled hashed area) is the $B_{s}^{0}$ signal component of the fit. The black horizontal lines guide the eye to better see the raw asymmetry of the signal strengths between particle and anti-particle decays.

To select beauty and charm events at LHCb in some cases cut based selections are applied, but most analyses use multivariate analysis methods like boosted decision trees. Typical separating variables, or their $\chi^{2}$, include track quality, particle identification (RICH, ECAL, MUON) for tracks, secondary vertex (SV) quality, kinematic variables like $p_{\mathrm{T}}$ of the candidate or daughter tracks, separation between primary vertex (PV) and SV, impact parameter of daughter tracks and the pointing of the candidate to the PV.

The remainder of these proceedings is organised as follows. In Sect. 2 examples of time integrated $C P$ violation measurements in $B$ meson systems at $\mathrm{LHCb}$ are described, followed by Sect. 3 that covers time dependent analyses. Section 4 covers $C P$ violation measurements in charm decays and Sect. 5 closes the paper with some conclusions.

\section{Time integrated measurements in the $B$ systems}

\section{1 $C P$ violation in $B^{0} \rightarrow K^{+} \pi^{-}$and $B_{s}^{0} \rightarrow K^{-} \pi^{+}$}

For the decay modes $B^{0} \rightarrow K^{+} \pi^{-}$and $B_{s}^{0} \rightarrow K^{-} \pi^{+}$, as described in Sect. 1, the asymmetries $A_{\text {raw }}=$ $\frac{N_{\tilde{f}^{-}}-N_{f}}{N_{\tilde{f}}+N_{f}}$ for $f=K^{+} \pi^{-}\left(B^{0}\right), K^{-} \pi^{+}\left(B_{s}^{0}\right)$ have been measured [12]. For this analysis $1 \mathrm{fb}^{-1}$ of data have been used. Due to interference between a penguin diagram and a tree diagram (i.e., one without loops) $C P$ violation is expected and also NP contributions are possible in the penguin loop.

Figure 6 shows the resulting invariant mass plots which reveal a raw asymmetry for $B^{0}$ as well as $B_{s}^{0}$. To get the true $C P$ asymmetry, the raw asymmetry has to be corrected for two effects. The detection asymmetry $A_{\mathrm{D}}$ is corrected using $D^{*+} \rightarrow D^{0}\left(K^{-} \pi^{+}\right) \pi^{+}$and $D^{*+} \rightarrow D^{0}\left(K^{-} K^{+}\right) \pi^{+}$decays which must show the same kind of asymmetry. The usage of the second of these decay modes is necessary to disentangle the unknown production asymmetry of $D^{*+}$ mesons. The production asymmetry of the beauty mesons on the other hand is corrected using the time dependence of the raw asymmetry $A_{\text {raw }}(t) \approx A_{C P}+A_{\mathrm{D}}+A_{\mathrm{P}} \cos (\Delta t)$ which is shown in Fig. 7. The largest systematic errors are the detection asymmetry in the case of $B^{0}$ and the signal model in case of $B_{s}^{0}$.

The $B^{0}$ result is given by

$$
A_{C P}\left(B^{0} \rightarrow K^{+} \pi^{-}\right)=-0.080 \pm 0.007 \text { (stat.) } \pm 0.003 \text { (syst.) }
$$



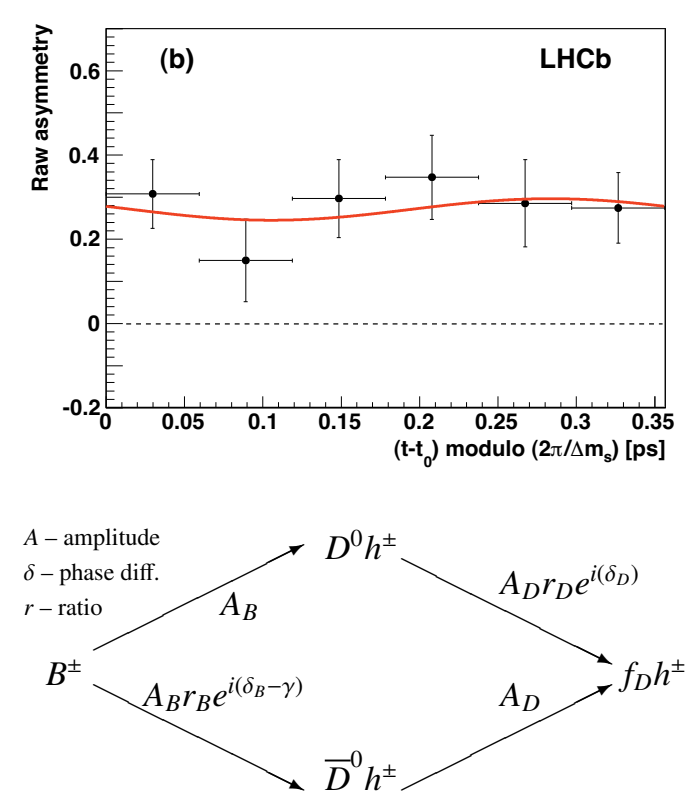

Figure 7. Time dependence of the raw asymmetry for $B_{s}^{0} \rightarrow K^{-} \pi^{+}$[12].
Figure 8. A diagram representing the decays of $B^{ \pm} \rightarrow D^{0} h^{ \pm}$and $B^{ \pm} \rightarrow \bar{D}^{0} h^{ \pm}$and how they can interfere. In course of the decay chain different phases are picked up depending on the path. This phase difference contains the CKM angle $\gamma$.

in agreement with the world average of $-0.098 \pm 0.013$ [29] and being the most precise measurement of this observable to date. While the result on $B_{s}^{0}$ gives

$$
A_{C P}\left(B_{s}^{0} \rightarrow K^{-} \pi^{+}\right)=0.27 \pm 0.04 \text { (stat.) } \pm 0.01 \text { (syst.) }
$$

which is the first observation $(6.5 \sigma)$ of $C P$ violation in a $B_{s}^{0}$ decay.

These measurements can also be used to test the SM prediction [30]

$$
\Delta=\frac{A_{C P}\left(B^{0} \rightarrow K^{+} \pi^{-}\right)}{A_{C P}\left(B_{s}^{0} \rightarrow K^{-} \pi^{-}\right)}+\frac{B R\left(B_{s}^{0} \rightarrow K^{-} \pi^{-}\right)}{B R\left(B^{0} \rightarrow K^{+} \pi^{-}\right)} \frac{\tau_{d}}{\tau_{s}}=0,
$$

where $B R$ denotes the branching fraction and $\tau_{d / s}$ is the mean life time of the $B_{d / s}^{0}$ meson. From the measurements we get $\Delta=-0.02 \pm 0.05 \pm 0.04$ consistent with the SM.

\subsection{Determination of the CKM angle $\gamma$ from trees}

As mentioned in the introduction, the unitary triangle angle $\gamma$ is the least well measured parameter of the CKM-matrix and thus crucial to measure in order to probe the unitarity condition on the CKMmatrix in the SM. To measure $\gamma$ from decays that only involve tree diagrams has the advantage that the theory error in the measurement is very small, of the order of $\delta \gamma / \gamma<O\left(10^{-6}\right)$ [31]. In other words we are here very sure that we really measure $\gamma$ with only very minor higher order effects. To do this we use decays of the kind $B^{ \pm} \rightarrow D^{0} h^{ \pm}$and $B^{ \pm} \rightarrow \bar{D}^{0} h^{ \pm}$with different $D^{0}$ decay modes, where the final state can be the same for both, as illustrated in Fig. 8. The interference of $b \rightarrow c$ and $b \rightarrow u$ decays gives sensitivity to $\gamma$ with direct $C P$ violation possible, but not necessary.

There are three different main approaches named by the first letters of the names of their proponents. The first one is the GLW method $[32,33]$ that uses $C P$ eigenstates, here $f_{D}=K^{+} K^{-}, \pi^{+} \pi^{-}$. The second one is the ADS method [34] where Cabibbo favoured, as well as a doubly Cabibbo suppressed 

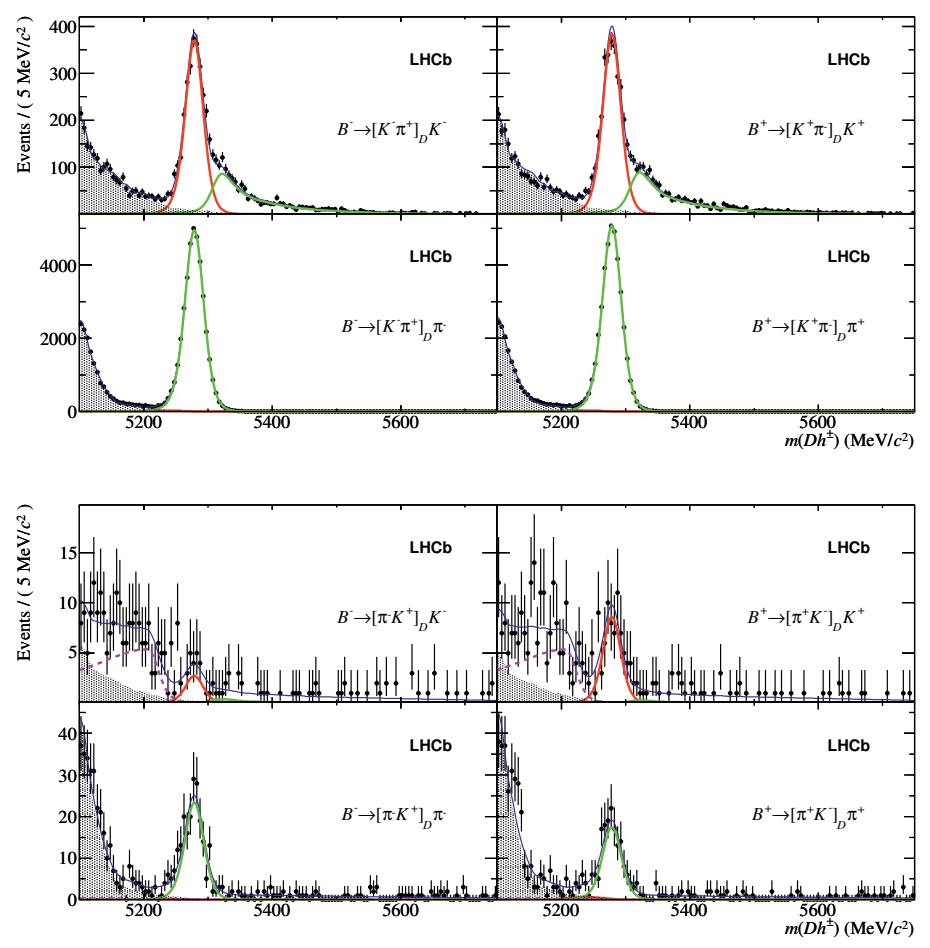

Figure 9. Example mass plots for the ADS method [36]. Shown is the Cabibbo favoured decay $D^{0} \rightarrow K^{-} \pi^{+}$, i.e., where the involved CKM matrix elements are large. The red line is the fit component due to the $B$ decay involving a kaon in the final state, while the green line is the fit component due to the $B$ decay having a pion in the final state.

Figure 10. Example mass plots for the ADS method [36], as Fig. 9, but for the doubly Cabibbo suppressed decay $D^{0} \rightarrow \pi^{-} K^{+}$that involves two small CKM matrix elements. An asymmetry between particle and anti-particle production due to direct $C P$ violation can be seen.

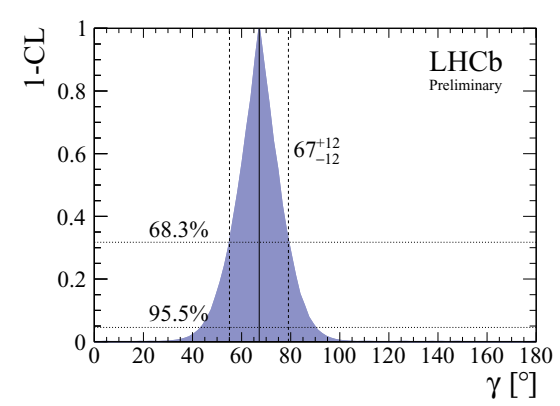

Figure 11. Confidence level plot for the CKM angle $\gamma$ from the combination of different methods [28]. For details please refer to the text.

decay are used, here $f_{D}=K^{\mp} \pi^{ \pm}$and $f_{D}=K^{\mp} \pi^{ \pm} \pi^{+} \pi^{-}$. The third of these methods is called GGSZ [35] and uses a Dalitz plot analysis, here with the decays $B^{ \pm} \rightarrow D K^{ \pm}$and $f_{D}=K_{\mathrm{s}}^{0} \pi^{+} \pi^{-}, K_{\mathrm{s}}^{0} K^{+} K^{-}$.

These methods have been used on $1 \mathrm{fb}^{-1}$ of data for the ADS and GLW method and on $3 \mathrm{fb}^{-1}$ for the GGSZ method and some sample mass plots are given in Figs. 9 and 10. The results have been combined in Ref. [28]. The resulting confidence level plot is shown in Fig. 11 and the numerical result is

$$
\gamma=(67 \pm 12)^{\circ} \text { at } 68 \% \mathrm{CL}, \text { modulo } 180^{\circ} \text { (preliminary) }
$$

This is in agreement with results from the experiments $\operatorname{BaBar}\left(\gamma=(69 \pm 17)^{\circ}\right.$ [37] $)$ and Belle $\left(\gamma=(68 \pm 14)^{\circ}\right.$ [37] $)$, but has smaller errors. It also agrees with a prediction from a fill fit to the CKM-unitary triangle data excluding the direct $\gamma$ measurements: $\gamma=\left(69.7_{-2.8}^{+1.3}\right)^{\circ}$ [37]. 


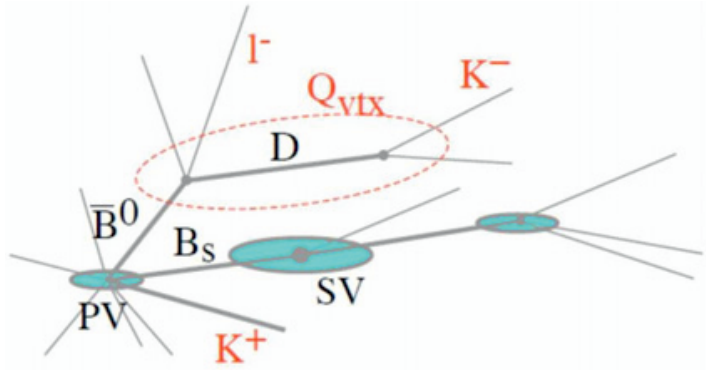

Figure 12. Sketch of the principles of flavour tagging in the example for a $B_{s}^{0}$ that is produced in a $p p$-collision at the $\mathrm{PV}$ and decays at the $\mathrm{SV}$. As described in the text, to deduce its flavour at production, the flavour of the other $B$ hadron, a $\bar{B}^{0}$ in this case, can be deduced, e.g., by the charges of its final state particles (upper part in red). In addition, the charge of the other strange hadron from the PV, a $K^{+}$in the example (red), can also be used.

\section{Time dependent measurements in the $B$ systems}

In this section some examples of time dependent analyses at $\mathrm{LHCb}$ in $B$ systems are described. In each case $1 \mathrm{fb}^{-1}$ of data has been used. For these analyses the interference between mixing and decay is exploited as explained in Sect. 1. To do this the flavour (i.e., whether it is a particle or an antiparticle) of the particle at production has to be known. The technique to do this on a statistical basis is called flavour tagging. A simplified example of this procedure is given in Fig. 12. The flavour of the produced particle can be tagged by the flavour of the opposite side $B$ hadron (as $b$ quarks are produced in $b \bar{b}$ pairs, there is always a flavour anti-correlated second $B$ hadron), or in the case of $B_{s}^{0}$ that contains an $s$ quark, by the charge of the strange hardon that originates from the hadronisation of the other strange quark, as strange quarks also come in $s \bar{s}$ pairs. For more details on flavour tagging at $\mathrm{LHCb}$ please refer to Ref. [38].

As the oscillations of $B_{s}^{0}$ are very fast, the decay time resolution is very important. Typical $B_{s}^{0}$ decay time resolutions at $\mathrm{LHCb}$ are $\sigma_{t} \sim 40$ to $50 \mathrm{fs}$, which is much smaller than the oscillation period $\frac{2 \pi}{\Delta m_{s}}=355 \mathrm{fs}$ [39]. In the case of decays into two vector particles there is an additional complication, i.e., an angular decomposition is needed to disentangle the $C P$ eigenstates of the final state. Thus a fit to the full time dependent terms is necessary and not just a fit to the asymmetry.

\subsection{Time dependent analysis of $B_{s}^{0} \rightarrow K^{+} K^{-}$}

The decay $B_{s}^{0} \rightarrow K^{+} K^{-}$has two pseudoscalar particles in the final state, thus the asymmetry can be used. This $C P$ asymmetry is of theoretical value, as combined with that of $B^{0} \rightarrow \pi^{+} \pi^{-}$it can be used to measure the CKM angle $\gamma$ in decays that include penguin diagrams [40]. This in turn can then be compared to the results from $\gamma$ from trees (see Sect. 2.2) and any differences would be a sign for NP.

The invariant mass plot and the oscillation of this measurement [6] are shown in Fig. 13. The largest systematic error is the decay time resolution width. The numerical results are

$$
\begin{aligned}
C_{K K}= & -0.14 \pm 0.11 \text { (stat) } \pm 0.03 \text { (syst), } \\
S_{K K}= & -0.30 \pm 0.12 \text { (stat) } \pm 0.04 \text { (syst), } \\
\rho\left(C_{K K}, S_{K K}\right)= & 0.02,
\end{aligned}
$$

where $\rho$ is the correlation. The significance for $\left(C_{K K}, S_{K K}\right)$ to differ from $(0,0)$ is $2.6 \sigma$. The measurement is still statistically limited and thus there are good prospects for future measurements.

\subsection{Time dependent analysis of $B_{s}^{0} \rightarrow \phi \phi$}

The decay $B_{s}^{0} \rightarrow \phi \phi$ is also called the golden penguin mode for decays into two vector particles as it has a very small $C P$ phase in the $\mathrm{SM} \phi_{s}^{\phi \phi}=\phi_{\mathrm{m}}-2 \phi_{\mathrm{D}} \approx 0.01 \mathrm{rad}$. Thus it is a null test of the SM, i.e., 

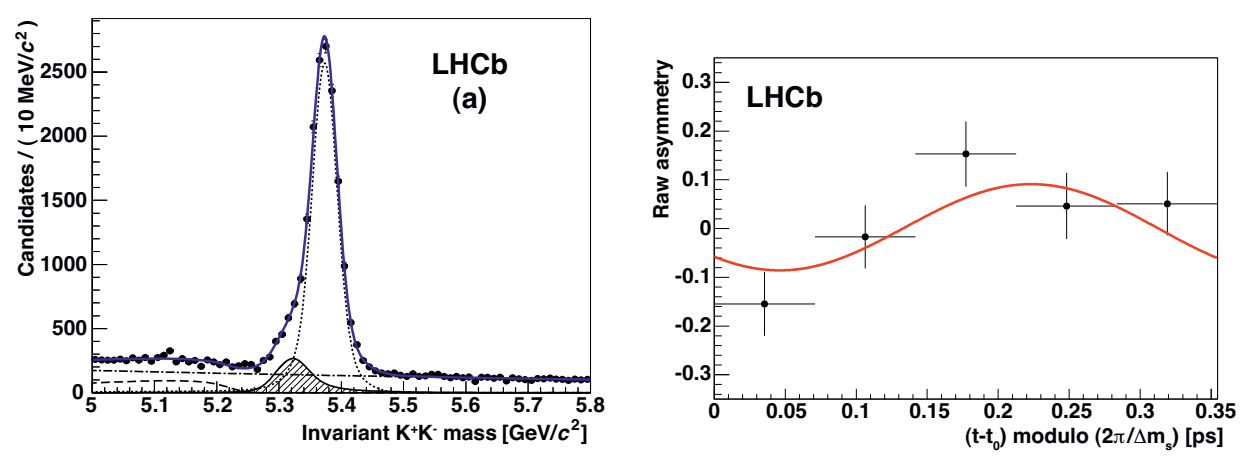

Figure 13. Invariant mass (left) of $B_{s}^{0} \rightarrow K^{+} K^{-}$and time evolution of the asymmetry (modulo one oscillation period) in the invariant mass around the signal peak (right). Both are taken from Ref. [6].
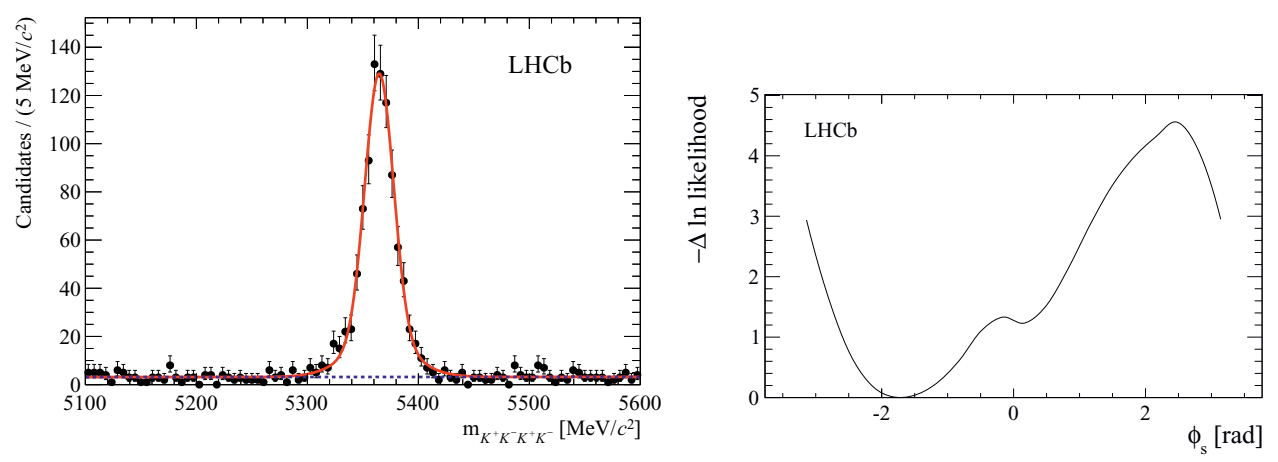

Figure 14. Invariant mass (left) of $B_{s}^{0} \rightarrow \phi \phi$ and negative $\log$ likelihood scan of $\phi_{s}^{\phi \phi}$ shifted vertically such that the minimum is at $-\Delta \ln$ likelihood $=0$ (right). Both are taken from Ref. [13].

even small contributions from NP can show up in the measurement. The invariant mass distribution and the likelihood scan over the parameter $\phi_{s}^{\phi \phi}$ of the analysis by LHCb [13] are given in Fig. 14. The largest systematic error is a possible $\mathrm{S}$-wave (i.e., scalar particle) contribution. The numerical result is

$$
\phi_{s}^{\phi \phi} \in[-2.46,-0.76] \quad \operatorname{rad} \text { at } 68 \% \mathrm{CL} .
$$

The result is still statistically limited, so future measurements using more data will give improved results.

\subsection{Measurement of $\phi_{s}$ in $B_{s}^{0} \rightarrow J / \psi h^{+} h^{-}$}

The decay $B_{s}^{0} \rightarrow J / \psi h^{+} h^{-}$is the golden mode for NP in $C P$ violation in $B_{s}^{0}$. It also has a small $C P$ phase in the SM of $\phi_{s}=-2 \beta_{s}=(-0.036 \pm 0.002) \mathrm{rad}$ [41], where $\beta_{s}$ is an angle in one of the (non-standard) unitary triangles. So this is also a null test of the SM. In our analysis [42] two decay modes are used. Firstly the mode $B_{s}^{0} \rightarrow J / \psi K^{+} K^{-}$which happens mainly through $B_{s}^{0} \rightarrow J / \psi \phi$ 
EPJ Web of Conferences

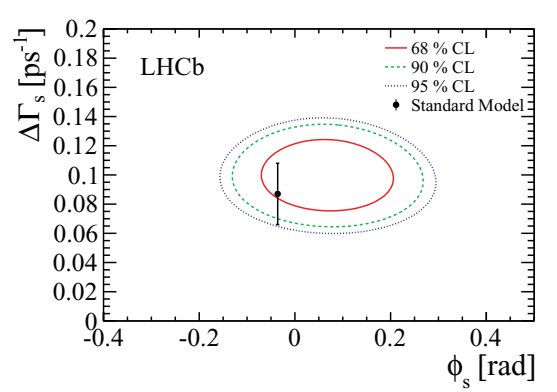

Figure 15. Confidence contours in $\phi_{s}$ and $\Delta \Gamma_{s}$ for the $B_{s}^{0} \rightarrow J / \psi K^{+} K^{-}$mode [42].
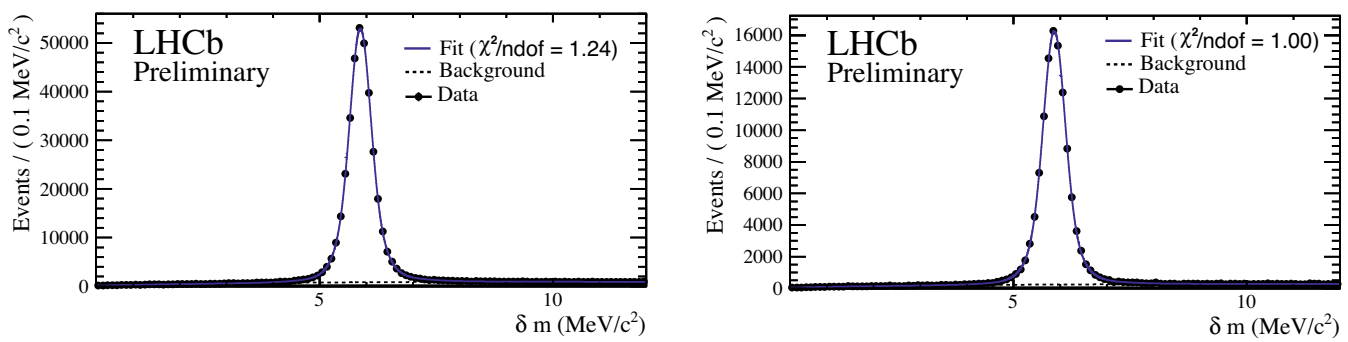

Figure 16. Difference of the invariant masses of the $D^{*+}$ and the $D^{0}, \delta m=m\left(D^{* \pm}\right)-m\left(D^{0}\right)$, for $D^{0} \rightarrow K^{+} K^{-}$ (left) and $D^{0} \rightarrow \pi^{+} \pi^{-}$(right) in the prompt analysis [8].

has a larger branching fraction, but an angular decomposition is needed to separate $C P$ components. Secondly the $B_{s}^{0} \rightarrow J / \psi \pi^{+} \pi^{-}$decay which happens mainly through $B_{s}^{0} \rightarrow J / \psi f_{0}(980)$ has a smaller branching fraction, but no angular decomposition is needed as $f_{0}(980)$ is a scalar particle. The result on $B_{s}^{0} \rightarrow J / \psi K^{+} K^{-}$is the most precise measurement of $\phi_{s}, \Gamma_{s}$ and $\Delta \Gamma_{s}$ to date and is depicted in Fig. 15.

The combined result for $B_{s}^{0} \rightarrow J / \psi h^{+} h^{-}$are given by

$$
\begin{aligned}
\phi_{s} & =0.01 \pm 0.07 \text { (stat) } \pm 0.01 \text { (syst) } \mathrm{rad}, \\
\Gamma_{s} & =0.661 \pm 0.004(\text { stat }) \pm 0.006(\text { syst }) \mathrm{ps}^{-1}, \\
\Delta \Gamma_{s} & =0.106 \pm 0.011(\text { stat }) \pm 0.007(\text { syst }) \mathrm{ps}^{-1} .
\end{aligned}
$$

This is in good agreement with the SM.

\section{Search for $C P$ violation in $D$ systems}

In this section we restrict ourselves to the results on the search for direct $C P$ violation in $D^{0} \rightarrow$ $h^{+} h^{-}[7,8]$. As the $D^{0}$ oscillation is rather slow, the $D^{0}$ mainly decays before it mixes. Thus time integrated measurements mainly yield direct $C P$ violation, unless $C P$ violation from mixing is very strong.

The raw asymmetry here is given by $A_{\text {raw }}=\frac{N\left(D^{0} \rightarrow f\right)-N\left(\bar{D}^{0} \rightarrow f\right)}{N\left(D^{0} \rightarrow f\right)+N\left(\bar{D}^{0} \rightarrow f\right)}$. To make the production and detection asymmetries cancel to first order, we construct the variable $\Delta A_{C P}=A_{C P}\left(K^{+} K^{-}\right)-A_{C P}\left(\pi^{+} \pi^{-}\right) \approx$ $A_{\text {raw }}\left(K^{+} K^{-}\right)-A_{\text {raw }}\left(\pi^{+} \pi^{-}\right)$. We use two different flavour tagging methods, the prompt one and the secondary one. In the prompt analysis the pion charge in $D^{*+} \rightarrow D^{0} \pi^{+}$versus $D^{*-} \rightarrow \bar{D}^{0} \pi^{-}$reveals 
the flavour of the $D^{0}$, while in the secondary analysis it is the charge of the muon in $\bar{B} \rightarrow D^{0} \mu^{-} X$ versus $B \rightarrow \bar{D}^{0} \mu^{+} X$ (where $X$ stands for any number of extra particles). The invariant mass plots for the prompt analysis are shown in Fig. 16.

The results are

$$
\begin{aligned}
\text { prompt: } & \Delta A_{C P}=(-0.34 \pm 0.15(\text { stat }) \pm 0.10(\text { syst })) \%[8] \\
\text { secondary: } & \Delta A_{C P}=(+0.49 \pm 0.30(\text { stat }) \pm 0.14(\text { syst })) \%[7],
\end{aligned}
$$

with the largest systematic errors, which can decrease with the use of more data, being the soft pion multiple scattering in case of the prompt analysis and a low lifetime background in $D^{0} \rightarrow \pi^{+} \pi^{-}$in the secondary case. The two results are compatible at $3 \% \mathrm{CL}$. The LHCb average gives $\Delta A_{C P}=(-0.15 \pm$ $0.16) \%$ (preliminary) which is compatible with the world average $\Delta a_{C P}^{\mathrm{dir}}=(-0.33 \pm 0.12) \%$ [43]. Here $\Delta A_{C P}=\Delta a_{C P}^{\mathrm{dir}}+\frac{\Delta\langle t\rangle}{\tau} a_{C P}^{\text {ind }}$, where $\Delta\langle t\rangle$ is the difference between the two decays' mean decay time in the sample and $\frac{\Delta\langle t\rangle}{\tau} \approx 11 \%$ for the prompt analysis and $\frac{\Delta\langle t\rangle}{\tau} \approx 2 \%$ in the secondary analysis respectively. The indirect $C P$ violation parameter $a_{C P}^{\text {ind }}$ has been measured to be well below one [43].

Thus there is only a hint for $C P$ violation in charm and there is a debate on whether such a relatively large $C P$ violation in charm would be possible in the SM or not. All other $C P$ violation searches in charm yielded negative results.

\section{Conclusions}

We have shown that $C P$ violation and flavour physics are powerful tools to find deviations from the $\mathrm{SM}$. LHCb has performed many world leading results on $C P$ violation in charm and beauty systems. The first observation of $C P$ violation in a $B_{s}^{0}$ decay has been achieved, but no deviation from the SM has been observed so far. Especially $\phi_{s}$ in $B_{s}^{0} \rightarrow J / \psi h^{+} h^{-}$has been, and still is, a good candidate for finding NP. It is now measured to very good precision in accordance with the SM, reducing the possible phase space of NP models. Most published analyses of LHCb use only one third of the data that is now available. In addition LHCb will resume data taking in 2015 and will upgrade the detector in 2018. So there is still large room to find NP at LHCb in near or far future.

\section{References}

[1] M. Beyer, ed., CP Violation in Particles, Nuclear and Astrophysics, Lecture Notes in Physics (Springer Verlag Berlin Heidelberg, 2002)

[2] C. Jarlskog, ed., CP Violation, Advanced Series on Directions in High Energy Physics (World Scientific Publishing Co. Pte. Ltd., 1989)

[3] R. Aaij et al. (LHCb Collaboration), New J. Phys. 15, 053021. 18 p (2013)

[4] A.A. Alves et al. (LHCb), JINST 3, S08005 (2008)

[5] R. Aaij et al. (LHCb Collaboration), Phys. Rev. D 85, 091105 (2012)

[6] R. Aaij et al. (LHCb collaboration), Tech. Rep. arXiv:1308.1428. CERN-PH-EP-2013-144. LHCB-PAPER-2013-040, CERN, Geneva (2013)

[7] R. Aaij et al. (LHCb Collaboration), Phys. Lett. B 723, 33 (2013)

[8] LHCb (LHCb Collaboration), LHCb-CONF-2013-003 (2013)

[9] R. Aaij et al. (LHCb collaboration), Phys. Lett. B 726, 646 (2013)

[10] R. Aaij et al. (LHCb Collaboration), Phys. Lett. B 721, 24 (2012)

[11] R. Aaij et al. (LHCb Collaboration), J. High Energy Phys. 06, 112. 22 p (2013) 
[12] R. Aaij et al. (LHCb Collaboration), Phys. Rev. Lett. 110, 221601. 9 p (2013)

[13] R. Aaij et al. (LHCb Collaboration), Phys. Rev. Lett. 110, 241802. 15 p (2013)

[14] R. Aaij et al. (LHCb Collaboration), Phys. Rev. Lett. 110, 031801. 16 p (2012)

[15] R. Aaij et al. (LHCb Collaboration), Phys. Rev. D 87, 112010. 39 p (2013)

[16] R. Aaij et al. (LHCb collaboration), Phys. Lett. B 726, 623 (2013)

[17] R. Aaij et al. (LHCb collaboration), Phys. Rev. Lett. 111, 151801. 16 p (2013)

[18] LHCb (LHCb Collaboration), LHCb-CONF-2012-029 (2012)

[19] R. Aaij et al. (LHCb collaboration), Phys. Rev. D 88, 052002. 22 p (2013)

[20] R. Aaij et al. (LHCb Collaboration), Phys. Rev. D 84, 112008. 13 p (2011)

[21] R. Aaij et al. (LHCb Collaboration), Nucl. Phys. B 867, 1 (2012)

[22] LHCb (LHCb Collaboration), LHCb-CONF-2013-009 (2013)

[23] R. Aaij et al. (LHCb Collaboration), Phys. Lett. B 718, 43 (2012)

[24] R. Aaij et al. (LHCb collaboration), Phys. Rev. Lett. 111, 101801. 9 p (2013)

[25] R. Aaij et al. (LHCb collaboration), Phys. Rev. D 88, 052015. 20 p (2013)

[26] R. Aaij et al. (LHCb collaboration), Tech. Rep. arXiv:1308.1048. LHCB-PAPER-2013-033. CERN-PH-EP-2013-141, CERN, Geneva (2013), submitted to Phys. Lett. B

[27] R. Aaij et al. (LHCb Collaboration), J. High Energy Phys. 03, 067. 16 p (2012)

[28] LHCb (LHCb Collaboration), LHCb-CONF-2013-006 (2013)

[29] Y. Amhis et al. (Heavy Flavor Averaging Group (HFAG)), arXiv:1207.1158 (2012)

[30] H.J. Lipkin, Physics Letters B 621, 126 (2005), hep-ph/0503022

[31] J. Brod, J. Zupan, ArXiv e-prints (2013), 1308. 5663

[32] M. Gronau, D. London, Physics Letters B 253, 483 (1991)

[33] M. Gronau, D. Wyler, Physics Letters B 265, 172 (1991)

[34] D. Atwood, I. Dunietz, A. Soni, Phys. Rev. Lett. 78, 3257 (1997)

[35] A. Giri, Y. Grossman, A. Soffer, J. Zupan, Phys. Rev. D 68, 054018 (2003)

[36] R. Aaij et al. (LHCb Collaboration), Phys. Lett. B 712, 203 (2012)

[37] The CKMfitter group (2013), http://ckmfitter.in2p3.fr/www/results/plots_fpcp13/ckm_res_fpcp13.html

[38] R. Aaij et al. (LHCb Collaboration), Eur. Phys. J. C 72, 2022. 28 p (2012)

[39] J. Behringer et al. (Particle Data Group), Phys. Rev. D 86, 010001 (2012)

[40] R. Fleischer, Physics Letters B 459, 306 (1999)

[41] J. Charles, O. Deschamps, S. Descotes-Genon, R. Itoh, H. Lacker, A. Menzel, S. Monteil, V. Niess, J. Ocariz, J. Orloff et al. (The CKMfitter Group), Phys. Rev. D 84, 033005 (2011)

[42] R. Aaij et al. (LHCb Collaboration), Phys. Rev. D 87, 112010. 39 p (2013)

[43] HFAG Group, results on CP violation in charm (2013), http://www.slac.stanford.edu/xorg/hfag/charm/index.html 